\title{
Un projet politique majeur
}

La rénovation de la formation professionnelle au Maroc

A key policy challenge. Modernising vocational training in Morocco

Un proyecto político mayor. La renovación de la formación profesional en

Marruecos

\section{Zayer Elmajid}

\section{(2) OpenEdition}

\section{Journals}

Édition électronique

URL : https://journals.openedition.org/ries/1638

DOI : 10.4000/ries. 1638

ISSN : 2261-4265

Éditeur

France Education international

Édition imprimée

Date de publication : 1 décembre 2003

Pagination : 63-72

ISBN : 978-2-8542-0558-9

ISSN : $1254-4590$

\section{Référence électronique}

Zayer Elmajid, « Un projet politique majeur », Revue internationale d'éducation de Sèvres [En ligne], 34

décembre 2003, mis en ligne le 22 novembre 2011, consulté le 07 juillet 2021. URL : http://

journals.openedition.org/ries/1638; DOI : https://doi.org/10.4000/ries.1638 


\section{Un projet politique majeur}

\section{La rénovation de la formation professionnelle au Maroc}

\section{Zayer Elmajid}

La formation professionnelle au Maroc a connu de multiples changements et réformes depuis sa création et son organisation moderne au temps du protectorat, c'est-à-dire depuis la création de la première école industrielle au Maroc en 1917.

En effet, on est passé des écoles techniques aux collèges techniques pour finir par les lycées techniques et les établissements de la formation professionnelle. Si les lycées techniques permettent de préparer le baccalauréat pour accéder soit à l'enseignement supérieur, soit aux filières de BTS ${ }^{1}$ (professionnelles), les établissements de la formation professionnelle sont très diversifiés par leurs modes de fonctionnement, leurs programmes et diplômes selon qu'ils relèvent de tel département ministériel ou de tel autre. Longtemps, les différents secteurs administratifs ont cherché à former leur propre personnel. Le système a certainement permis de répondre aux besoins importants de la période précédant l'indépendance.

Aujourd'hui il s'essouffle et la tendance est à l'optimisation des moyens et des structures, d'où un retour progressif vers un pilotage unique permettant de dégager les économies en ressources humaines et matérielles préconisées par la Charte de l'éducation et de la formation.

Conscientes des énormes efforts qui attendent le Maroc en matière de développement socio-économique et de la place qu'occupe le développement, de la promotion et la formation des ressources humaines dans ce processus, les plus hautes autorités du Royaume du Maroc ont inscrit la réforme du système d'éducation et de formation comme deuxième priorité nationale.

\section{LA RÉFORME DU SYSTÈME DE FORMATION PROFESSIONNELLE: UNE PRIORITÉ NATIONALE}

Face à la mondialisation des marchés et pour répondre à une demande de plus en plus grande née de la massification de l'appareil d'éducation, le système de formation professionnelle doit relever plusieurs défis.

Il doit aider l'entreprise marocaine à faire face aux contraintes de la compétitivité, à se mettre à niveau et à moderniser ses moyens et modes de production. Pour cela, il doit adapter ses programmes et ses prestations aux besoins de l'entreprise en matière de formation initiale et continue. 
La formation professionnelle étant un système conçu pour être ouvert et attentif à l'évolution économique et technologique, les stratégies d'amélioration et d'adéquation permettront de renforcer et d'affiner les liens avec les différents secteurs d'activité économique, de répondre aux besoins en compétences des entreprises et de favoriser l'insertion des diplômés.

Pour ce faire, l'optimisation de l'offre de formation, l'amélioration de la qualité de la formation par la certification et la réforme des modes de formations (initiale, alternée, cours du soir, formation insertion) s'avèrent nécessaires. Il s'agit en fait de répondre quantitativement et qualitativement aux besoins en compétences des acteurs économiques.

Lors de son discours de politique générale devant le parlement, le Premier ministre a dévoilé le 21 novembre 2002 le programme du gouvernement en matière de formation professionnelle.

Il a annoncé que son gouvernement prévoit le doublement des effectifs de la formation professionnelle qui devra profiter à 400000 jeunes au cours du prochain quinquennat, tandis que le programme formation insertion sera développé au profit de 20 à 25000 diplômés par an.

\section{LES STRUCTURES DE PILOTAGE}

Si l'on met de côté les formations spécialisées relevant de certains départements ministériels telles que les professions de santé, d'agriculture, de l'armée, du tourisme, de police ou encore les formations de personnels pour l'éducation nationale, l'essentiel du système de formation professionnelle est géré par trois départements: l'Éducation nationale pour les formations de BTS domiciliées dans les lycées et les ENSET, le ministère de l'Enseignement supérieur, de la formation des cadres et de la recherche scientifique pour les DUT délivrés par les écoles supérieures de technologie (EST) et enfin le secrétariat d'État à la formation professionnelle par le biais de l'Office de la formation professionnelle et de la promotion du travail (OFPPT) qui est de loin le premier opérateur de formation professionnelle au Maroc.

\section{L'Office de la formation professionnelle et de la promotion du travail (OFPPT)}

Créé en 1974, l'OFPPT est un organisme doté de la personnalité morale et de l'autonomie financière. Sa gestion est tripartite et fait intervenir des représentants des employeurs, des salariés et de l'État. Il est administré par un conseil présidé par le ministre en charge de la formation professionnelle.

L'office est chargé depuis sa création de développer la formation professionnelle et de l'adapter aux besoins de l'économie nationale.

Ainsi, l'OFPPT s'est engagé à former une main-d'œuvre qualifiée, à favoriser la promotion sociale des travailleurs et à adapter la formation aux besoins du marché du travail. 
Dès 1984, date de la réforme du système de la formation professionnelle, l'OFPPT a connu un important développement et s'est trouvé investi de nouvelles missions: favoriser la prise de conscience du rôle déterminant du facteur humain dans le développement et la compétitivité de l'entreprise d'une part, associer les employeurs aux processus de formation par une participation directe aux différents cursus de formation d'autre part.

Avec ses modes de formation diversifiés, ses prestations adaptées aux besoins des entreprises, son implantation nationale et l'implication sans cesse croissante des professionnels, la formation professionnelle s'est forgée une position de choix dans le contexte socio-économique du Maroc.

L'Office comptait, en 2002, dix directions régionales, 185 établissements de formation avec 192 spécialités au profit de 57300 stagiaires en formation initiale, 22000 participants en cours du soir, 92140 salariés en formation continue. L'ensemble était encadré par 5881 collaborateurs (formateurs et personnels administratifs). Par ailleurs, l'Office développe quatre niveaux de formation, du plus bas au plus élevé: niveau spécialisation, niveau qualification, niveau technicien, niveau technicien spécialisé.

Conditions d'accès à la formation

\begin{tabular}{|c|c|c|}
\hline Niveau & Conditions d'accès & Observations \\
\hline $\begin{array}{l}\text { Technicien } \\
\text { spécialisé }\end{array}$ & $\begin{array}{l}\text { Être âgé de } 23 \text { ans } \\
\text { et être titulaire du baccalauréat }\end{array}$ & $\begin{array}{l}\text { Les candidats issus des branches économiques } \\
\text { ou littéraires ne peuvent accéder qu'aux filières } \\
\text { de gestion des entreprises, commerce international, } \\
\text { secrétariat de direction } \\
\text { Par contre, les candidats issus des branches scientifiques } \\
\text { et techniques industrielles peuvent s'inscrire } \\
\text { dans toutes les filières offertes }\end{array}$ \\
\hline Technicien & $\begin{array}{l}\text { Être âgé de moins de } 25 \text { ans } \\
\text { et avoir au moins } \\
\text { le niveau baccalauréat }\end{array}$ & $\begin{array}{l}\text { Les candidats issus des branches scientifiques } \\
\text { et techniques industrielles peuvent s'inscrire } \\
\text { dans toutes les filières. Par contre, les candidats issus } \\
\text { des branches littéraires ne peuvent s'inscrire } \\
\text { qu'en gestion des entreprises, secrétariat de direction, } \\
\text { comptabilité, techniques d'habillement, } \\
\text { techniques de production }\end{array}$ \\
\hline Qualification & $\begin{array}{l}\text { Être âgé de moins de } 25 \text { ans et } \\
\text { avoir atteint le niveau } 9^{\mathrm{e}} \text { année } \\
\text { de l'enseignement fondamental } \\
\text { (équivalent de la } 3^{\mathrm{e}} \\
\text { dans le système français), } \\
1^{\text {re }} \text { ou } 2^{\mathrm{e}} \text { année secondaire }\end{array}$ & \\
\hline Spécialisation & $\begin{array}{l}\text { Être âgé de } 15 \text { à } 30 \text { ans } \\
\text { et avoir atteint le niveau } \\
6^{\mathrm{e}}, 7^{\mathrm{e}} \text { ou } 8^{\mathrm{e}} \text { année } \\
\text { de l'enseignement fondamental, } \\
\text { ou CAP }\end{array}$ & \\
\hline
\end{tabular}




\section{Impliquer les professionnels dans le processus de formation}

Dans un souci permanent d'adéquation «formation/emploi», l'OFPPT attache une importance particulière à l'implication soutenue des professionnels dans le processus de formation et d'insertion.

C'est ainsi que sont directement impliquées les associations et les fédérations professionnelles. Leur implication grandissante s'effectue dans un esprit de partenariat étroit et induit des évolutions dans trois directions: la diversification des modes de formation, la croissance des capacités d'accueil et des effectifs, la préoccupation permanente de la qualité des produits et services de l'Office, mesurée notamment par la recherche d'une meilleure adéquation aux besoins effectifs des entreprises.

Ainsi de nombreuses initiatives sont lancées pour satisfaire la demande des entreprises avec la qualité requise: mise en place des schémas directeurs de la formation professionnelle à l'échelon régional pour apporter localement les réponses appropriées; mise en place de nouvelles dispositions pour la formation professionnelle alternée, la formation des adultes, l'apprentissage et les cours du soir; évaluation du système d'orientation/sélection des candidats; nouveau processus d'évaluation des acquis des stagiaires et généralisation progressive d'évaluation de l'encadrement des examens de fin de formation par un jury professionnel; mise à l'étude de la réforme des cursus pédagogiques.

\section{Accroître la capacité d'accueil}

L'évolution de la capacité d'accueil et des effectifs nécessite de la part de l'Office des efforts en matière d'optimisation des ressources matérielles et humaines de manière à répondre à l'accroissement des besoins de plus en plus diversifiés et des demandes de formation de plus en plus nombreuses de la part des jeunes.

À la rentrée 2003-2004, 55103 places pédagogiques sont offertes pour un effectif total de stagiaires supérieur à 92000 . Ceci ne va pas sans poser quelques problèmes en ce qui concerne le recrutement puisqu'en moyenne l'Office reçoit plus de cinq demandes pour une place pédagogique, voire dix pour le niveau technicien spécialisé.

Toujours en collaboration étroite avec les associations professionnelles, l'Office prépare les nouvelles formations qui répondront à leurs besoins. Seront en particulier créateurs d'emplois les secteurs de l'agroalimentaire, des nouvelles technologies de l'information et de la communication (NTIC), du tourisme (hôtellerie et restauration), de la plasturgie.

\section{Améliorer la qualité de la formation}

Conscient de l'importance du niveau et de la qualité de formation des formateurs, les responsables de l'Office ont pris la mesure de l'enjeu: la révision des programmes est validée par des équipes pédagogiques issues de l'enseignement technique ainsi que par des agrégés et les formateurs se perfectionnent grâce à 
des formations techniques, des stages en milieu professionnel, des formations portant sur la pédagogie et sur l'ingénierie de formation sans oublier les actions de renforcement des compétences et de certification. La démarche qualité de l'Office comporte ainsi cinq composantes stratégiques: la qualité de recrutement des formateurs; le développement du professionnalisme des formateurs; l'évaluation du cursus de formation, la restructuration des formations; la professionnalisation des prestations de l'Office.

La formation initiale comprend une formation théorique générale et spécialisée, une formation technique pratique et des stages en milieu professionnel. Les formateurs effectuent un service de vingt-quatre heures hebdomadaires de formation. Ils peuvent être ingénieurs ou diplômés de l'enseignement supérieur, recrutés parmi les meilleurs diplômés de la formation professionnelle ou encore issus de l'entreprise, plus rarement des écoles normales supérieures de l'enseignement technique (ENSET).

\section{Une politique active de coopération internationale}

Pour suivre au plus près l'évolution technologique et pouvoir répondre aux besoins du marché national et international, l'Office a mis en œuvre depuis longtemps une politique active de coopération.

De fructueuses coopérations bilatérales sont en cours avec l'Allemagne, le Royaume de Belgique et la communauté française de Belgique, couvrant de larges domaines qui comprennent aussi bien des stages dans ces pays auprès d'organismes appropriés, que la mise à disposition d'experts, d'une assistance technique, de formateurs et de différentes formes d'appui (documentaire, financement de certains aspects de la mise à niveau du dispositif de l'Office).

La coopération avec la France s'effectue en collaboration avec l'AFPA ${ }^{1}$ dans le cadre de la mise en œuvre du programme AMO (accords de maind'œuvre) qui prévoit des stages, de l'expertise et de la formation, ainsi qu'avec l'Agence Française de développement pour la création de quatre nouveaux ISTA (institut supérieurs de technologie appliquée).

D'autres coopérations sont développées avec l'Espagne, l'Italie, les Pays-Bas, le Japon, la Corée du Sud, les pays arabes, les pays d'Afrique subsaharienne et l'Europe Centrale.

La coopération avec le Canada apporte un appui important au développement du dispositif de l'Office dans le cadre du programme PRICAM (programme de renforcement institutionnel entre le Canada et le Maroc) ainsi qu'un concours du fonds de développement du secteur privé au Maroc.

De nombreuses coopérations multilatérales sont également développées avec l'Union européenne, notamment dans le cadre du programme MEDA,

1. AFPA: association nationale pour la formation professionnelle des adultes. 
ainsi qu'avec la Banque mondiale (BIRD) pour un troisième projet de développement du secteur privé qui porte notamment sur les centres d'excellence et les contrats spéciaux de formation.

\section{Le ministère de l'Éducation nationale}

\section{Repositionner l'enseignement technique}

Avant le développement de la formation professionnelle, l'enseignement secondaire technique pouvait remplir deux missions: préparer les jeunes soit à intégrer le marché du travail soit à poursuivre des études supérieures (dont l'accès était très limité à l'époque). Aujourd'hui, avec un enseignement technique supérieur plus important, qu'il soit court (de type bac +2 ) ou long (maîtrise ou classes préparatoires +3 années), l'enseignement secondaire technique a presque abandonné la finalité de préparer les jeunes à intégrer le marché du travail et privilégie surtout la poursuite des études. Il développe en son sein depuis 1992 des formations de type de brevet de techniciens supérieurs qui sont des formations professionnelles par excellence.

Les succès rencontrés auprès des employeurs dès la sortie des premières promotions ont servi de déclencheurs d'un processus continu de développement de ces formations.

Ainsi, on est passé de six BTS avec six spécialités dans quatre centres en 1992 à quarante-huit BTS avec vingt-trois spécialités dans vingt-neuf autres centres. Aujourd'hui, le système permet la mise sur le marché chaque année d'environ mille lauréats de BTS toutes spécialités confondues.

Ces formations, qui sont domiciliées pour la plupart dans des lycées techniques, sont ouvertes d'abord aux titulaires du baccalauréat de l'enseignement technique. Quelques filières permettent néanmoins l'accès aux autres types de baccalauréat.

Les programmes des BTS marocains sont inspirés de leurs équivalents français. Certains ont été bâtis avec l'appui et la coopération française, d'autres tout simplement copiés parfois sans les précautions d'usage et donc sans respecter le processus de construction tel qu'il est suivi en France.

Ces formations, bien qu'elles bénéficient de certaines conditions favorables, doivent à court terme être révisées et leur mode de management réadapté pour qu'elles puissent continuer à bénéficier des faveurs des employeurs.

En effet, les titulaires d'un BTS sont issus d'un enseignement technique secondaire dispensé en français et bénéficient ainsi d'un vécu de cinq années d'enseignement et de formation technique dans la langue usuelle de l'entreprise marocaine.

De plus, ces formations bénéficient d'un encadrement de haut niveau par des professeurs agrégés des différentes disciplines scientifiques et techniques. 
Par contre, étant domiciliées dans des lycées techniques, elles ne disposent pas de l'autonomie morale et financière. De plus, les personnels de direction de ces établissements, y compris les chefs de travaux n'ont pas ou presque pas de relations avec le monde socio-économique. Des dispositions doivent être prises à ce niveau pour développer ces relations ne serait ce que pour organiser les stages en entreprise.

L'enseignement technique secondaire qui représente à peine $5 \%$ de l'enseignement secondaire au Maroc se maintient au gré des réformes. Il a bénéficié ces dernières années d'un appui constant de l'inspection générale française à travers les différents programmes de coopération bilatérale. Il s'apprête à connaître un développement notable durant les cinq prochaines années avec le doublement de ces effectifs dans le cadre d'un programme de développement (de construction et d'équipement de vingt établissements) financé par la Banque africaine de développement (BAD). La réforme de l'éducation et de la formation l'oblige à développer des synergies avec les autres systèmes de formation et surtout lui assigne la mission de domicilier un enseignement professionnel débouchant sur un baccalauréat professionnel.

\section{Le ministère de l'Enseignement supérieur}

Le ministère de l'Enseignement supérieur pilote à travers les universités les écoles supérieures de technologie (sur le modèle des IUT en France) qui délivrent le DUT. Ces formations sont similaires à celles développées en France et connaissent le même succès. Toutefois, les lauréats issus de ces écoles optent aujourd'hui davantage pour des études supérieures type "maîtrise» dans les facultés des sciences et techniques ou les écoles d'ingénieurs.

La réforme du système d'éducation et de formation au Maroc verra l'avènement d'un enseignement professionnel supérieur dès le premier cycle universitaire. Cette réforme va démarrer dès la rentrée universitaire 2003/2004. Ce type d'enseignement aspire à drainer au moins $50 \%$ de la population des étudiants qui intègrent l'université.

Les EST continuent de bénéficier d'une coopération soutenue et concertée avec la France et l'Union européenne.

\section{La Charte nationale D'ÉDUCATION ET DE FORMATION}

Le Royaume du Maroc a initié début 1999 une réflexion collective approfondie sur le devenir de son système d'éducation et de formation.

Menée par une commission spéciale éducation-formation (COSEF), composée de représentants de tous les partis politiques et syndicats siégeant au Parlement, de dignitaires religieux, d'organisations non gouvernementales, d'experts, d'opérateurs économiques et de personnalités de la société civile, cette réflexion 
à débouché en juillet 1999 sur la production d'un document intitulé Charte nationale d'éducation et de formation.

Le document est divisé en deux parties: la première partie énonce les fondements et les principes sur lesquels se base la nouvelle vision de l'école présentée par la Charte; la deuxième partie fixe des échéances, des objectifs et des modes d'organisation et de gestion du système d'éducation formation à travers six espaces: espace 1, extension de l'enseignement et de son ancrage à l'environnement économique; espace 2, organisation pédagogique; espace 3 : amélioration de la qualité de l'éducation et de la formation; espace 4 : ressources humaines; espace 5 : gouvernance; espace 6 : partenariat et financement.

En octobre 1999, le roi Mohammed VI a validé les résultats des travaux de cette commission et invité le gouvernement et le parlement à la mise en ouvre de la Charte nationale d'éducation-formation dans toutes ses composantes.

Les premières lois d'application sont votées par le parlement dès mars et les différents autres projets de lois et de décrets ont été mis en chantier sitôt après et votés depuis.

Au cours de l'été 2000, le roi déclare l'éducation et la formation deuxième priorité nationale après l'intégrité territoriale et la décennie 2000-2010, décennie nationale de l'éducation-formation. L'année scolaire 2000-2001 en constituait le premier exercice scolaire et universitaire.

Parmi les objectifs de la Charte, nous ne retenons dans ce document que ceux qui intéressent la formation professionnelle. Ainsi, dans son levier $\mathrm{n}^{\circ} 3$ intitulé "adéquation du système d'éducation et de formation à son environnement économique», la Charte prévoit:

- la mise en place progressive des réseaux d'éducation et de formation dès la rentrée 2005 afin d'optimiser les ressources et les moyens;

- une proportion de $50 \%$ des personnes professionnellement qualifiées arrivant chaque année sur le marché du travail à l'horizon 2010 (actuellement 20\%);

- une formation par apprentissage de 10000 jeunes à partir de 2000;

- une formation par apprentissage de 50000 jeunes à partir de 2005;

- une formation alternée de 12000 jeunes à partir de 2000 ;

- une formation alternée de 30000 jeunes à partir de 2005;

- l'orientation vers les branches scientifiques, techniques et professionnelles des deux tiers de l'effectif total de l'enseignement secondaire et supérieur.

\section{Les objectifs à moyen terme (2000-2011)}

Les objectifs fixés pour le développement de la formation professionnelle peuvent se décliner comme suit:

- développement de l'appareil de formation, dont les effectifs globaux des stagiaires, tous modes de formation confondus, passeront de 149600 en 2000 à 247400 en 2005 et à 360600 en 2011 ; 
- développement de la formation par apprentissage dans la perspective de faire bénéficier de ce mode de formation 40000 personnes à l'horizon 2004 et 60000 en 2010 contre 3600 en 2000 ;

- mise à niveau et promotion de la formation professionnelle privée pour accueillir 80000 stagiaires en 2005 et 124000 en 2011, contre 56000 en 2000;

- extension de la formation alternée dans l'objectif de faire bénéficier de ce mode de formation 14000 stagiaires dans le secteur public de formation professionnelle à l'horizon 2005 contre 9600 en 2000;

- mise sur le marché du travail de 123700 personnes diplômées ou qualifiées en 2005 et 173000 à l'horizon 2011 contre 72000 en 2000;

- généralisation de l'approche par compétences pour l'élaboration et la maintenance des programmes de formation;

- mise à niveau des ressources formatives humaines et valorisation du vécu professionnel des formateurs afin de constituer progressivement un corps ayant le vécu et la culture de l'entreprise;

- doublement des effectifs des lauréats des sections de techniciens supérieurs ;

- mise en place du baccalauréat technico-professionnel;

- ouverture de passerelles aux diplômés de la formation professionnelle pour leur permettre de préparer ce cycle de baccalauréat et par la suite les brevets de techniciens supérieurs voire l'université;

- mise en place du premier cycle universitaire comportant une option sanctionnée par un diplôme d'enseignement universitaire professionnel (DEUP) débouchant directement sur la vie active;

- harmonisation de l'évaluation finale de telle sorte que le diplôme délivré soit le même en tout point du territoire.

\section{LES ATTENTES EN MATIÈRE DE COOPÉRATION INTERNATIONALE}

Désormais, le marché est international, la technologie est internationale. La formation professionnelle doit s'inscrire dans cette logique. Qu'il s'agisse de la production de biens ou de services, ils doivent, pour être monnayables répondre aux normes et aux standards reconnus internationalement. Les différentes réformes en cours qui touchent la réforme des programmes selon la nouvelle approche par compétences, la mise à niveau des ressources humaines et matérielles et la promotion d'un nouveau management du système de la formation professionnelle doivent aboutir, à terme, à une certification garante de l'adoption d'une assurance qualité.

L'implication de la coopération internationale dans les différents chantiers et projets permettra de garder le cap et de coller le plus près possible aux changements nés de l'évolution des marchés, des technologies et des comportements humains. 
Le système technico-professionnel marocain est et a toujours été intimement lié au modèle français. De plus, la France demeure le premier partenaire économique et commercial du Maroc. Rien de plus naturel que de voir ces liens se renforcer. La langue française, qui reste la langue véhiculaire dans ce système, doit servir de vecteur pour renforcer ce développement.

Le point nodal de cette coopération internationale et franco-marocaine doit être la formation et la mise à niveau des formateurs sans oublier les formateurs de formateurs.

\section{Perspectives}

Le système aspire, une fois les différentes réformes abouties, à plus de sérénité pour s'occuper de l'optimisation de toutes les autres formations professionnelles sectorielles. On tendrait ainsi vers une meilleure utilisation de toutes les ressources. Les retombées pourraient être multiples. On ne citera que le produit de la taxe professionnelle qui ne bénéficie aujourd'hui qu'aux seuls établissements dépendant de l'Office de la formation professionnelle et de la promotion du travail.

La mise en place d'un véritable système d'orientation, l'observation précise et fine du marché du travail, l'analyse appropriée des désiratas des jeunes conjugués avec la mise en place du baccalauréat et des premiers cycles universitaires professionnels constitueront autant d'atouts pour que le système soit performant.

Le soin particulier que le système doit réserver au choix et à la formation de ses formateurs est de nature à asseoir définitivement cette performance.

Les échanges internationaux, l'immersion dans le monde de l'entreprise constituent autant d'étapes importantes pour l'évolution des carrières.

Enfin, une remise en question périodique du dispositif s'avère nécessaire pour sa dynamique. Il doit se soumettre à des évaluations internes et externes et en tirer les enseignements nécessaires pour son évolution.

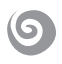

Les formations techniques et professionnelles au Maroc avaient longtemps vécu en marge d'un enseignement général pourvoyeur d'élites dirigeantes à tous les niveaux. En effet, les formations rencontraient un double handicap: le déficit d'image auprès d'une jeunesse assimilant les blouses bleues ou grises à une corvée durant toute leur vie et un coût élevé qui rebutait les décideurs les plus enclins à les développer.

La massification de l'éducation, le chômage et particulièrement celui des diplômés, la mondialisation enfin allaient se conjuguer quelques années plus tard pour faire prendre conscience aux responsables des enjeux qui attendent le Maroc et surtout de la nécessité de la mise à niveau de son économie. 
Cette dernière doit nécessairement passer également par la mise à niveau des ressources humaines et par conséquent du système d'éducation et de formation. C'est pourquoi la réforme de l'éducation formation a été placée deuxième priorité nationale et la décennie en cours déclarée décennie de l'éducation et de la formation.

Les chantiers actuels tendent tous vers des accroissements quantitatifs et qualitatifs de la formation professionnelle. Les maîtres mots sont: optimisation, diversification, compétence, qualité, orientation et insertion.

Les pouvoirs publics, à travers les budgets nationaux ou à travers des coopérations, bi ou multilatérales, consacrent ce rythme de développement en réservant à chaque fois une grosse part des financements à la formation.

L'objectif d'orienter les deux tiers des élèves vers les filières scientifiques et technico-professionnelles, le nombre de candidats garçons et filles de plus en plus élevé (parfois dix candidats pour une place offerte), désireux d'intégrer les filières professionnelles, le foisonnement des établissements d'enseignement et de formation privés, le développement des modes de formation sont autant d'atouts pour un système qui a longtemps été en quête de reconnaissance. Aux hommes et aux femmes concernés par le système de la formation professionnelle de rester vigilants pour que cette reconnaissance perdure. 